Based on observations made with the Télescope Bernard Lyot at Pic du Midi Observatory, France.

\title{
Speckle observations of composite spectrum stars with PISCO in 1993-1998.
}

\author{
J.-L. Prieur, L. Koechlin, N. Ginestet, J.-M. Carquillat
}

UMR 5572, Observatoire Midi-Pyrénées - CNRS, 14 Avenue E. Belin, 31400 Toulouse, France.

and

E. Aristidi

UMR 6525, Université de Nice Sophia-Antipolis - CNRS, Parc Valrose, 06108 Nice Cedex

2, France.

and

M. Scardia

Osservatorio Astronomico di Brera, Via E. Bianchi 46, 22055 Merate, Italy.

and

L. Arnold

Observatoire de Haute-Provence, 04870 St Michel l'Observatoire, France.

and

R. Avila

Instituto de Astronomia UNAM, Campus Morelia, A.P. 72-3 (Xangari), 58089 Morelia Michoacan, Mexico.

and

M.C. Festou

UMR 5572, Observatoire Midi-Pyrénées - CNRS, 14 Avenue E. Belin, 31400 Toulouse, France.

and

S. Morel

ESO, Karl Schwarzschild Str. 2, 85748 Garching bei München, Germany. 
and

J.-P. Pérez

UMR 5572, Observatoire Midi-Pyrénées - CNRS, 14 Avenue E. Belin, 31400 Toulouse, France.

\begin{abstract}
We present speckle interferometry observations of 47 composite spectrum stars obtained between 1993 and 1998 at the Pic du Midi Observatory with the PISCO speckle camera. $76 \%$ of over 150 independent 10 -min sequences of observations led to a companion detection. Binary component angular separations ranged from $0.05^{\prime \prime}$ to $1.2^{\prime \prime}$. We also obtained a series of 23 measurements of an additional 9 close binaries. PISCO observations confirm, for the first time since their discovery, the duplicity of HD 29104 (L 4), HD 83808 (WGT 1Aa), HD 183912 Aa' (BON Ap), and HD 156729 (HR 6436). Discovered as double by Hipparcos, the particularly difficult to resolve HD 156729 was observed despite the large magnitude difference, $\Delta m=4.2$, between its two components.
\end{abstract}

Subject headings: Techniques: interferometric — Astrometry — Stars: binaries: close, visual — Stars: composite spectrum

\title{
1. Introduction
}

Composite spectrum stars are binary stars (often spectroscopic binary stars) comprising in most cases a dwarf hot star ( $\mathrm{B}$ or A-type) and a cool evolved star ( $\mathrm{G}, \mathrm{K}$ or $\mathrm{M}$ ) (Ginestet et al. 1992). The study of these systems is of great astrophysical interest (i) to improve estimates of the mass of red giant stars, and (ii) to constraint stellar evolution models (Schröder et al. 1997). A major problem arises when it comes to identifying the spectral types of the two components. As speckle interferometry can resolve some of these objects, we have undertaken an observational program with PISCO $^{1}$ at the Pic du Midi Observatory and we report here on the astrometry done on some of them. The photometry and the spectra of the individual components will be presented elsewhere (Prieur et al., in preparation).

In 1993, we established the list of our targets from the following sources:

- Hynek's catalogue of composite spectrum stars (Hynek 1938),

- McAlister's catalogue of interferometric binaries (38),

${ }^{1}$ PISCO stands for "Pupil Interferometry Speckle camera and COronagraph". 
- Griffin's list of spectroscopic binaries where he suggests that most of them are likely to be resolved with speckle interferometry (Griffin 1990),

- a list of stars with composite spectra from Markowitz (1969).

Additional objects suspected to be composite stars - e.g., stars with abnormal color indices in the Bright Star Catalogue (Hoffleit \& Jaschek, 1982) - have also been incorporated to our list.

When starting this project, most of the objects had already been resolved by speckle interferometry but their orbits were undetermined. To obtain a good determination of the spectral types of the two components, two of us (N.G. and J.-M.C.) have carried out a spectroscopic survey of these binaries with the Carélec and Aurélie spectrographs at the Haute-Provence Observatory. The results concerning the cool components were presented in (Ginestet et al., 1997, 1999). The classification of the hot components is in progress (Ginestet \& Carquillat, in preparation).

During the 1993-1998 exploitation phase of PISCO, we applied a "flexible" scheduling to maximize the scientific return. Depending on the weather conditions, we selected the astrophysical program best suited for these conditions. Since part of our observations could be done when the FWHM seeing was larger than 1.2 arcsec, our program was often used as a backup for observations requiring good atmospheric conditions (e.g., high resolution imaging of complex or faint objects). As a consequence, multiple observations of our targets were performed during the 1993-1998 period, which permitted a follow-up of the astrometry over 6 years for some of them.

\section{Observations}

The observations (Table 1) were carried out with the PISCO speckle camera (44) developed at the Midi-Pyrénées Observatory and operated at the Cassegrain Focus of the 2-meter Bernard Lyot Telescope (TBL) at Pic du Midi. PISCO is a remotely controlled versatile instrument whose observing modes (e.g., imaging or spectroscopy) can be configured in real time. It constitutes a powerful tool for investigating the field of close binary stars, as demonstrated in previous works (Aristidi et al. 1999; 48; 45).

In this program, the PISCO speckle camera was used in its full pupil imaging mode. The atmospheric chromatic dispersion was corrected in real time with computer controlled Risley prisms. Except when the wind was strong and caused an elongation of the autocorrelation peaks (e.g. for HD 33883), these peaks were circular which is a good indication of the quality of the dispersion correction.

PISCO can make use of various detectors, as described in Prieur et al. (1998). The observations presented here were performed with three detectors: the CAR (Caméra à Anode Résistive or Ranicon detector), the ICCD (Intensified CCD with a micro-channel plate) and 
the PAPA (photon-counting detector, derived from the version described in Papaliolios et al., 1985).

In Table 1, the nights are qualified as "used" when we could open the dome; however the FWHM seeing was generally much poorer than the yearly average TBL value of $\sim 1.2^{\prime \prime}$. During the period 1993-1998, over one hundred nights were allocated to the projects studied with PISCO, with a usability rate of $33 \%$ that is significantly less than the average rate of $\sim 50 \%$ for this site.

\section{Data reduction}

The data reduction of a given observing sequence started with a detector-dependent pre-processing phase that generated an autocorrelation according to the method described in Worden (1977). The mean inter-correlation of two frames separated by a delay larger than the coherence time was subtracted from the mean autocorrelation of the elementary frames. This removed the background of the mean autocorrelation and nicely increased the contrast of the secondary peaks of binary stars. Astrometry parameters were then derived from this autocorrelation following the procedure described in Prieur et al. (2001).

\subsection{Calibration}

The orientation of the frames was calibrated by combining two methods: (i) by using the star tracks obtained by moving the telescope along the declination and right ascension axes, and (ii) by using wide pairs with slow orbital motion as "astrometric standards": HIP 1447 and HIP 4065 (Cuypers \& Seggewiss, 1999, Prieur et al, 2001). The resulting angular uncertainty was found to be $\pm 0.4^{\circ}$ and was included in the error on the position angle measurements displayed in Tables 3 and 4.

Likewise, the magnification scale was obtained both by the "astrometric standards" and by measuring a calibration grid placed at the entrance focal plane of PISCO. A value of $50.4 \pm 0.1 \mathrm{~m}$ was used for the focal length of the TBL, as determined by the Pic du Midi optics group. This grid was also used to measure and correct the geometric distortion.

\subsection{Pre-processing data collected with the CAR detector}

The CAR detector produces no frames, but a continuous flow of photon coordinates in chronological order. With this camera, the mean autocorrelation was computed by correlating each photon coordinate with the $\mathrm{N}$ others collected during a given time interval. For each photon, this time interval, dubbed "the sliding window", starts after the photon is detected and ends after a chosen "frame time" has elapsed. This "frame time" can be chosen and adjusted during off-line data processing. If the frame time is significantly larger 
Table 1. Observations

\begin{tabular}{ccccc}
\hline \hline \multicolumn{1}{c}{ Dates } & Detector & \multicolumn{2}{c}{ Nights } & Program \\
& & Attributed & Used & \\
& & & & \\
\hline Aug. 1993 & CAR & - & $\sim 1$ & Backup \\
Sept. 1994 & CAR & - & $<1$ & Backup \\
July 1995 & CAR & 8 & 4 & Main \\
Jan. 1997 & CAR & 5 & 2 & Main \\
" & ICCD & & 1 & $"$ \\
June 1997 & PAPA & - & $<1$ & Backup \\
Aug. 1998 & ICCD & 7 & 5 & Main \\
\hline
\end{tabular}

Table 2. Filter characteristics.

\begin{tabular}{ccc}
\hline \hline Name & $\begin{array}{c}\text { Central wavelength } \\
(\mathrm{nm})\end{array}$ & $\begin{array}{c}\text { Bandwidth } \\
(\mathrm{nm})\end{array}$ \\
\hline $\mathrm{B}$ & 447 & 47 \\
OIII & 501 & 11 \\
V & 530 & 57 \\
R & 644 & 70 \\
RL & 743 & 69 \\
I & 855 & 74 \\
\hline
\end{tabular}


than the coherence time $\tau_{c}$ of the atmospheric turbulence, noise is added to high resolution structures, and this prevents any detection of the companion. If the frame time is too small, the signal to noise ratio is degraded by photon noise, which may also impede companion detection.

To determine the optimum frame time, which maximizes the contrast of the secondary peaks in the autocorrelation function of the binary star, we computed from the same data a series of autocorrelations with frame times spanning the range 2-20 ms. Optimal values were 5-8 ms, which implies that the turbulence was fast $\left(\tau_{c}=3-5 \mathrm{~ms}\right)$.

Another important parameter of the reduction procedure was the spatial sampling factor (re-binning) of the autocorrelations. For each data set, a compromise had to be found since a large spatial sampling increases the signal-to-noise ratio (by reducing the photon noise), while decreasing the angular resolution. Hence, by providing a list of photo-events coordinates, the CAR detector offers a high flexibility and the possibility to determine during the data processing phase the optimum values for the frame time and the sampling factor.

Unfortunately, this detector presents two defects which had to be calibrated and corrected:

1) A small geometric distortion: the detector axes (i.e. those of the resistive anode) were not strictly perpendicular relative to each other (difference up to $2.5^{\circ}$ ), and at a different scale (up to $2 \%$ ). To correct this geometric distortion, two-dimensional polynoms were fitted each night to images of the scaling grid available in PISCO, and applied to the CAR data.

2) A hole in the center of the autocorrelation function commonly appears when using detectors coupled to a micro-channel plate. This hole is due to the electronic depletion of a channel that appears after the detection of a photo-event in that channel. This depletion can last a few milliseconds and is a limitation to working at high illuminations. We thus limited the illumination to $10^{4}$ photons/s by inserting neutral densities for bright objects $(\mathrm{V}<7)$. We also modified the sliding window, shifting it by one or several milliseconds towards the future. In such a situation, each photon is not correlated with those immediately following, but correlated only with other photons detected at a time when the microchannels have partially recovered from depletion. The "photon counting hole" effect mainly affects the photometric measurements. Although it can reduce the detectability of very close binaries, it does not introduce artefacts on the astrometric measurements.

\subsection{Pre-processing data collected with the ICCD detector}

The data from the ICCD were stored as frames in SVHS tapes with a rate of 50 frames/s. For the ICCD, the exposure time of elementary frames could take any value between $64 \mu s$ and $16 \mathrm{~ms}$, but the time interval between two successive frames was fixed $(20 \mathrm{~ms})$. This 
detector could not work in genuine photon counting mode and thus did not provide the coordinates of individual photo-events. A specially designed program (by JLP), controlled a video-tape recorder and a digitizing board, allowing a fully automatic processing of these tapes (Prieur et al., 2001).

\subsection{Pre-processing data collected with the PAPA detector}

The pre-processing phase of the data obtained with the PAPA detector is described in (Aristidi et al. 1999) and concerns here only a few measurements.

\subsection{Astrometric measurements}

The treatment of the mean autocorrelations was identical for all detectors. It consisted in accurately measuring the position of the secondary peaks of the binary stars, from which the position angles and angular separations of the components were derived. This was interactively done by a set of procedures described in Prieur et al. (2001).

\section{Results}

The astrometric measurements are displayed in Tables 3 and 4. The HD name is in Col. 1 and the corresponding WDS name (Washington Double Star Catalogue, http://ad.usno.navy.mil/wds/wds.html) is given in Col. 2. For each observing sequence, we give the epoch of observation (Col. 3) in Besselian years, the filter (Col. 4) (whose characteristics are listed in Table 2), the detector used (Col. 5), the position angle $\theta$ in degrees (Col. 6), relative to North increasing in the direction of increasing right ascension, and the angular separation $\rho$ in arcseconds (Col. 7).

In Cols. 8 and 9 are indicated the residuals $O-C$ (Observed minus Computed) for the objects with a known orbit, whose origin is given in Col. 10: HP64 (Hopmann, 1964), HP73 (Hopmann, 1973), HZ82 (Heintz, 1982), SC83 (Scardia, 1983), DC84 (Docobo \& Costa, 1984), HZ86 (Heintz, 1986), HK89 (Hartkopf et al., 1989), MS97 (Mason, 1997), BG99 (Balega et al., 1999), HK99 (Hartkopf, 1999), MS99 (Mason et al., 1999a), SJ99 (Soderhjelm, 1999), HK00 (Hartkopf \& Mason, 2000) OJ00 (Olevic \& Jovanovic, 2000), PB00 (Pourbaix, 2000), and SC00 (Scardia et al., 2000b).

In some cases (noted as "Marginal detection" in Col. 11), although the companion could be detected, the measurements are not given as they would be too uncertain to be of any use. Note that the $V$ and $R$ measurements of the position angle of HD 49618 have large errors; they are given between brackets. In Col. 11, we also indicate if the object is known to be a spectroscopic binary (SB) and, when applicable, give its orbital period.

The position angle $\theta$ of the companion was measured from the autocorrelation function, 
which leaves a $180^{\circ}$ ambiguity. When the signal-to-noise ratio was good enough, the restricted triple-correlation technique described in Aristidi et al. (1997a), or the image restoration with bispectral techniques (Prieur et al., 1991), allowed us to remove this ambiguity. This is noted with an asterisk in Col. 6. We note that for HD 8036, the brightest component in the $B$ image is at $180^{\circ}$ relative to that found in the $V$ and $R$ images.

To have a more comprehensive view of our program on composite spectrum stars, we have included in Table 3 two previously published measurements of HD 83808 and HD 186518 made with PISCO (references given in Col. 11). 
Table 3. Relative astrometric data (epoch, $\rho, \theta$, resp. in Col. 3, 6 and 7) of the composite spectrum stars. The position angles $\theta$ have a $180^{\circ}$ ambiguity except those marked with an asterisc for which triple correlation methods removed this ambiguity.

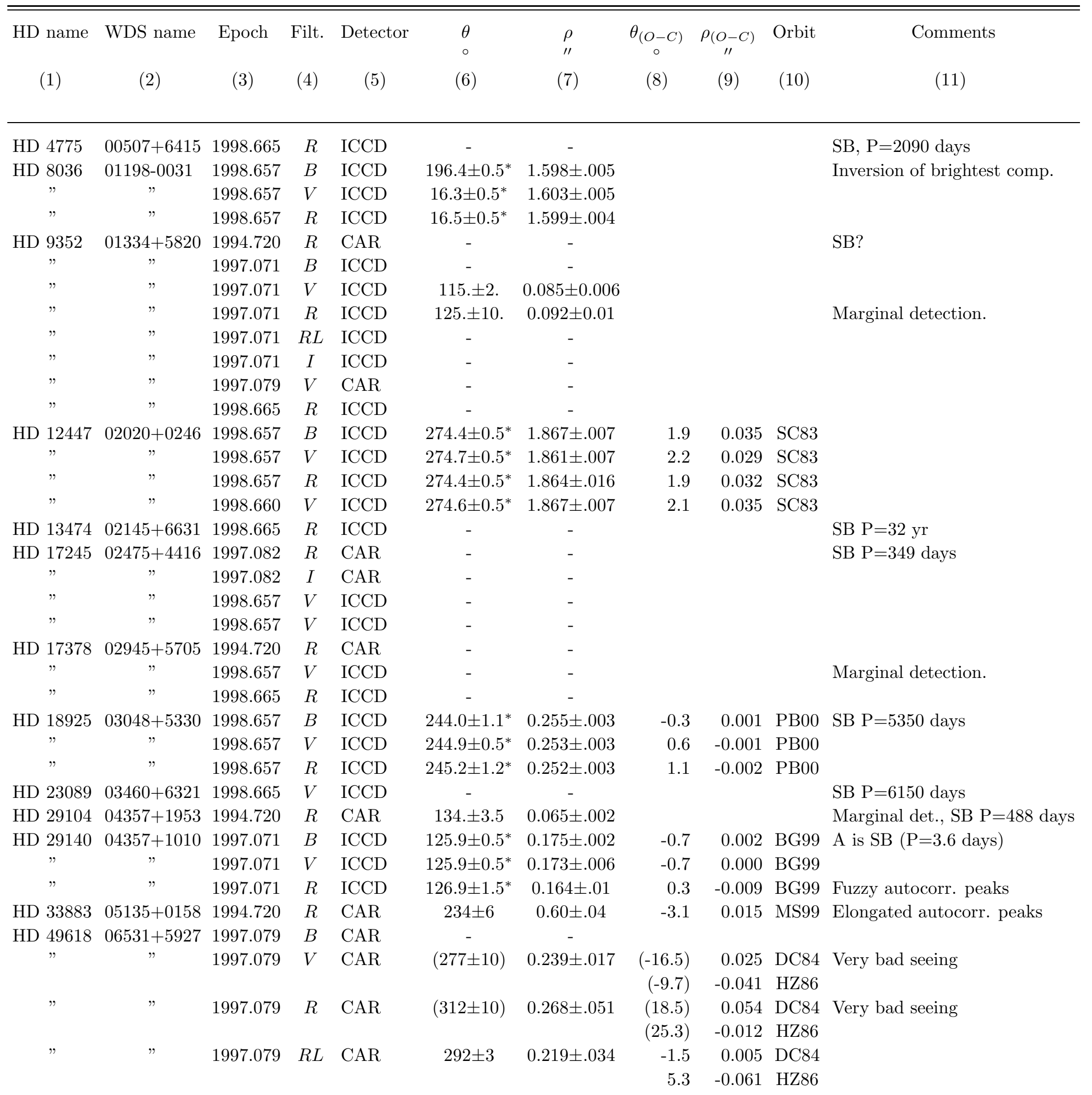


Table 3-Continued

\begin{tabular}{|c|c|c|c|c|c|c|c|c|c|c|}
\hline HD name & WDS name & $\begin{array}{c}\text { Epoch } \\
(3)\end{array}$ & Filt. & Detector & $\begin{array}{c}\theta \\
\circ \\
(6)\end{array}$ & $\begin{array}{c}\rho \\
\prime \prime \\
(7)\end{array}$ & $\begin{array}{c}\theta_{(O-C)} \\
\circ \\
(8)\end{array}$ & $\begin{array}{c}\rho_{(O-C)} \\
\quad " \\
(9)\end{array}$ & (10) & Comments \\
\hline$"$ & $"$ & 1997.079 & $I$ & CAR & $293 \pm 2$ & $0.240 \pm .027$ & $\begin{array}{r}-0.5 \\
6.3\end{array}$ & $\begin{array}{r}0.214 \\
-0.040\end{array}$ & $\begin{array}{l}\text { DC84 } \\
\text { HZ86 }\end{array}$ & \\
\hline HD 82072 & 09296-0307 & 1997.079 & $B$ & CAR & - & - & & & & \\
\hline$"$ & $"$ & 1997.079 & $R L$ & CAR & $30.3 \pm 0.9$ & $0.895 \pm .010$ & & & & \\
\hline$"$ & $"$ & 1997.082 & $B$ & CAR & $30.4 \pm 0.5$ & $0.868 \pm .024$ & & & & \\
\hline$"$ & $"$ & 1997.082 & $V$ & CAR & - & - & & & & \\
\hline$"$ & $"$ & 1997.082 & $R$ & CAR & - & - & & & & Marginal detection. \\
\hline HD 83808 & $09412+0954$ & 1997.071 & $V$ & ICCD & $267.9 \pm 0.5$ & $0.59 \pm 0.1$ & & & & (Aristidi et al., 1999) \\
\hline$"$ & $"$ & 1997.076 & $R$ & CAR & - & - & & & & $\mathrm{A}$ is $\mathrm{SB}, \mathrm{P}=14$ days \\
\hline$"$ & $"$ & 1997.079 & $R L$ & CAR & - & - & & & & \\
\hline$"$ & $"$ & 1997.079 & $I$ & CAR & - & - & & & & \\
\hline HD 85558 & 09525-0806 & 1997.082 & $B$ & CAR & $64.7 \pm 2$ & $0.594 \pm .038$ & 1.0 & -0.012 & HZ82 & \\
\hline$"$ & $"$ & 1997.082 & $V$ & CAR & - & - & & & & Marginal detection. \\
\hline$"$ & $"$ & 1997.082 & $R$ & CAR & $63.4 \pm 0.5$ & $0.615 \pm .013$ & -0.3 & 0.009 & HZ82 & \\
\hline HD 109358 & $12337+4121$ & 1997.079 & $V$ & CAR & - & - & & & & \\
\hline$"$ & $"$ & 1997.079 & $R$ & CAR & - & - & & & & \\
\hline HD 159870 & $17335+5734$ & 1995.556 & $B$ & CAR & $307.8 \pm 0.6$ & $0.099 \pm 0.005$ & & & & \\
\hline$"$ & $"$ & 1995.556 & $R$ & CAR & $308.1 \pm 0.7$ & $0.102 \pm 0.002$ & & & & \\
\hline$"$ & $"$ & 1995.561 & $V$ & CAR & $309.2 \pm 1.2$ & $0.094 \pm 0.004$ & & & & \\
\hline HD 166479 & $18101+1629$ & 1995.553 & $B$ & CAR & $220.1 \pm 0.5$ & $1.218 \pm 0.008$ & 1.7 & -0.023 & HP64 & \\
\hline$"$ & $"$ & 1995.553 & $V$ & CAR & $221.3 \pm 0.5^{*}$ & $1.221 \pm .019$ & 2.9 & -0.020 & HP64 & \\
\hline$"$ & $"$ & 1995.553 & $R$ & CAR & $220.4 \pm 0.5^{*}$ & $1.210 \pm .002$ & 2.0 & -0.031 & HP 64 & \\
\hline$"$ & $"$ & 1998.657 & $B$ & ICCD & $220.6 \pm 0.5^{*}$ & $1.228 \pm .002$ & 2.6 & -0.013 & HP64 & \\
\hline$"$ & $"$ & 1998.657 & $V$ & ICCD & $220.7 \pm 0.5^{*}$ & $1.225 \pm .002$ & 2.7 & -0.016 & HP64 & \\
\hline$"$ & $"$ & 1998.657 & $R$ & ICCD & $220.7 \pm 0.5^{*}$ & $1.229 \pm .002$ & 2.7 & -0.012 & HP64 & \\
\hline HD 169985 & $18272+0012$ & 1998.657 & $V$ & ICCD & - & - & & & & SB $\mathrm{P}=386$ d., triple. \\
\hline HD 178452 & $19082+1215$ & 1995.561 & $B$ & CAR & $181.9 \pm 1.5$ & $0.158 \pm .002$ & & & & \\
\hline$"$ & $"$ & 1995.561 & $V$ & CAR & $183.7 \pm 0.8$ & $0.156 \pm .004$ & & & & \\
\hline$"$ & $"$ & 1998.657 & $V$ & ICCD & $180.9 \pm 0.5$ & $0.169 \pm .01$ & & & & Weak autoc. peaks \\
\hline HD 183912 & $19307+2758 \mathrm{AB}$ & 1995.559 & $B$ & CAR & $234.3 \pm 1.0^{*}$ & $34.4 \pm 0.2$ & & & & Albireo \\
\hline$"$ & $"$ & 1995.559 & $V$ & CAR & $234.9 \pm 1.0^{*}$ & $34.0 \pm 0.2$ & & & & \\
\hline$"$ & $"$ & 1995.559 & $R$ & CAR & $233.2 \pm 1.0^{*}$ & $34.3 \pm 0.2$ & & & & \\
\hline HD 183912 & $19307+2758 \mathrm{Aa}$ & 1995.556 & $B$ & CAR & $139.0 \pm 0.8$ & $0.398 \pm 0.009$ & -1.9 & 0.008 & HK99 & \\
\hline$"$ & $"$ & 1995.556 & $R$ & CAR & $140.9 \pm 1.0$ & $0.397 \pm 0.009$ & -0.0 & 0.007 & HK99 & \\
\hline$"$ & $"$ & 1995.559 & $V$ & CAR & $139.3 \pm 1.0$ & $0.397 \pm .007$ & -1.6 & 0.007 & HK99 & \\
\hline$"$ & $"$ & 1998.657 & $B$ & ICCD & $132.7 \pm 0.5^{*}$ & $0.388 \pm .005$ & 0.5 & 0.018 & HK99 & \\
\hline$"$ & $"$ & 1998.657 & $V$ & ICCD & $132.7 \pm 0.5^{*}$ & $0.384 \pm .006$ & 0.5 & 0.007 & HK99 & \\
\hline$"$ & $"$ & 1998.657 & $R$ & ICCD & $132.3 \pm 0.9^{*}$ & $0.385 \pm .007$ & 0.9 & 0.008 & HK99 & \\
\hline$"$ & $"$ & 1998.660 & $V$ & ICCD & - & - & & & & \\
\hline HD 183912 & $19307+2758 \mathrm{Aa}^{\prime}$ & 1995.556 & $B$ & CAR & - & - & & & & Marginal detection. \\
\hline
\end{tabular}


Table 3-Continued

\begin{tabular}{|c|c|c|c|c|c|c|c|c|c|c|}
\hline HD name & WDS name & $\begin{array}{c}\text { Epoch } \\
(3)\end{array}$ & Filt. & Detector & $\begin{array}{c}\theta \\
\circ \\
(6)\end{array}$ & $\begin{array}{c}\rho \\
\prime \prime \\
(7)\end{array}$ & $\begin{array}{c}\theta_{(O-C)} \\
\circ \\
(8)\end{array}$ & $\begin{array}{c}\rho_{(O-C)} \\
\quad " \\
\quad(9)\end{array}$ & (10) & Comments \\
\hline$"$ & $"$ & 1995.556 & $R$ & CAR & - & - & & & & Marginal detection. \\
\hline$"$ & $"$ & 1995.559 & $V$ & CAR & $160.0 \pm 4.0$ & $0.045 \pm .003$ & & & & \\
\hline HD 184759 & $19348+2928$ & 1998.657 & $V$ & ICCD & - & - & & & & $\mathrm{SB} \mathrm{P}=1572$ days \\
\hline HD 186203 & $19426+1150$ & 1993.605 & $V$ & CAR & $73 . \pm 1$ & $0.39 \pm .01$ & & & & \\
\hline$"$ & $"$ & 1995.553 & $R$ & CAR & $76.4 \pm 1.0$ & $0.421 \pm 0.006$ & & & & \\
\hline$"$ & $"$ & 1995.559 & $B$ & CAR & $76.9 \pm 0.5$ & $0.430 \pm .005$ & & & & \\
\hline$"$ & $"$ & 1995.559 & $V$ & CAR & $76.7 \pm 0.5$ & $0.431 \pm .004$ & & & & \\
\hline$"$ & $"$ & 1998.657 & $B$ & ICCD & $77.9 \pm 0.5^{*}$ & $0.417 \pm .002$ & & & & \\
\hline$"$ & $"$ & 1998.657 & $V$ & ICCD & $77.4 \pm 0.5^{*}$ & $0.417 \pm .002$ & & & & \\
\hline$"$ & $"$ & 1998.657 & $R$ & ICCD & $77.0 \pm 0.5^{*}$ & $0.420 \pm .002$ & & & & \\
\hline HD 186518 & $19439+2708$ & 1998.657 & $B$ & ICCD & $282 . \pm 2$ & $0.348 \pm .02$ & & & & \\
\hline$"$ & $"$ & 1998.657 & $V$ & ICCD & $283.2 \pm 0.6$ & $0.360 \pm .003$ & & & & \\
\hline$"$ & $"$ & 1998.657 & $R$ & ICCD & $283.6 \pm 0.5^{*}$ & $0.349 \pm .004$ & & & & (Prieur et al., 2001) \\
\hline HD 187259 & $19487+1149$ & 1995.561 & $B$ & CAR & $106.6 \pm 0.7$ & $1.499 \pm .020$ & & & & \\
\hline$"$ & $"$ & 1995.561 & $V$ & CAR & $106.8 \pm 0.8$ & $1.451 \pm .008$ & & & & \\
\hline$"$ & $"$ & 1995.561 & $R$ & CAR & $107.6 \pm 0.8$ & $1.467 \pm .010$ & & & & \\
\hline$"$ & $"$ & 1998.657 & $B$ & ICCD & $107.3 \pm 0.5^{*}$ & $1.449 \pm .003$ & & & & \\
\hline$"$ & $"$ & 1998.657 & $V$ & ICCD & $107.4 \pm 0.5^{*}$ & $1.450 \pm .003$ & & & & \\
\hline$"$ & $"$ & 1998.657 & $R$ & ICCD & $107.1 \pm 0.5^{*}$ & $1.447 \pm .004$ & & & & \\
\hline$"$ & $"$ & 1998.660 & $V$ & ICCD & $107.0 \pm 0.5^{*}$ & $1.447 \pm .010$ & & & & \\
\hline HD 187321 & $19487+1852$ & 1998.657 & $B$ & ICCD & $100 . \pm 2 .^{*}$ & $0.400 \pm .02$ & & & & Artefact in central line \\
\hline$"$ & $"$ & 1998.657 & $V$ & ICCD & $99.5 \pm 0.5^{*}$ & $0.412 \pm .002$ & & & & \\
\hline$"$ & $"$ & 1998.657 & $R$ & $\mathrm{ICCD}$ & $99.6 \pm 0.5^{*}$ & $0.411 \pm .002$ & & & & \\
\hline HD 192577 & $20136+4644$ & 1998.665 & $V$ & ICCD & - & - & & & & $\mathrm{SB} \mathrm{P}=3784$ days \\
\hline$"$ & $"$ & 1998.665 & $R$ & ICCD & - & - & & & & \\
\hline HD 192909 & $20155+4743$ & 1998.665 & $R$ & ICCD & - & - & & & & $\mathrm{SB} P=1148$ days \\
\hline HD 194359 & $20244+2417$ & 1994.706 & OIII & CAR & $111.5 \pm 1.7$ & $0.363 \pm .004$ & & & & Noisy \\
\hline$"$ & $"$ & 1998.657 & $B$ & ICCD & $116.4 \pm 0.6^{*}$ & $0.353 \pm .004$ & & & & \\
\hline$"$ & $"$ & 1998.657 & $V$ & ICCD & $117.0 \pm 0.5^{*}$ & $0.357 \pm .004$ & & & & \\
\hline$"$ & $"$ & 1998.657 & $R$ & ICCD & $116.4 \pm 0.5^{*}$ & $0.352 \pm .002$ & & & & \\
\hline HD 195692 & $20320+2548$ & 1995.550 & $V$ & CAR & - & - & & & & $\mathrm{A}$ is $\mathrm{SB}(\mathrm{P}=11$ days $)$ \\
\hline$"$ & $"$ & 1995.550 & $R$ & CAR & $240.1 \pm 1.1$ & $0.161 \pm .005$ & & & & \\
\hline$"$ & $"$ & 1998.657 & $B$ & ICCD & $244.3 \pm 0.5$ & $0.223 \pm .002$ & & & & \\
\hline$"$ & $"$ & 1998.657 & $V$ & ICCD & $243.7 \pm 0.7$ & $0.226 \pm .006$ & & & & \\
\hline$"$ & $"$ & 1998.657 & $R$ & ICCD & $242.6 \pm 0.8^{*}$ & $0.227 \pm .006$ & & & & \\
\hline$"$ & $"$ & 1998.660 & $V$ & ICCD & $244.0 \pm 1.2^{*}$ & $0.225 \pm .008$ & & & & \\
\hline HD 196088 & $20330+4950$ & 1998.665 & $R$ & ICCD & $220.0 \pm 0.7$ & $0.082 \pm .003$ & 7.9 & 0.065 & OJ00 & SB \\
\hline HD 196093 & $20339+3515$ & 1994.706 & $V$ & CAR & - & - & & & & \\
\hline$"$ & $"$ & 1995.550 & $R$ & CAR & - & - & & & & \\
\hline$"$ & $"$ & 1998.657 & $B$ & ICCD & $280 . \pm 2$ & $0.317 \pm .04$ & & & & Artefact in central line \\
\hline
\end{tabular}


Table 3-Continued

\begin{tabular}{|c|c|c|c|c|c|c|c|c|c|c|}
\hline HD name & WDS name & (3) & Filt. & Detector & $\begin{array}{c}\theta \\
\circ \\
(6)\end{array}$ & $\begin{array}{c}\rho \\
\prime \prime \\
(7)\end{array}$ & $\begin{array}{c}\theta_{(O-C)} \\
\circ \\
(8)\end{array}$ & $\begin{array}{c}\rho_{(O-C)} \\
\quad " \\
\quad(9)\end{array}$ & (10) & Comments \\
\hline$"$ & $"$ & 1998.657 & $V$ & ICCD & - & - & & & & Marginal detection. \\
\hline$"$ & $"$ & 1998.657 & $R$ & ICCD & $278.4 \pm 1.5^{*}$ & $0.282 \pm .01$ & & & & \\
\hline HD 197177 & $20410+3218$ & 1998.657 & $V$ & ICCD & - & - & & & & \\
\hline HD 199306 & $20537+5918$ & 1998.665 & $R$ & ICCD & $97.4 \pm 0.5$ & $0.166 \pm .002$ & -0.1 & -0.001 & HK89 & \\
\hline HD 203338 & $21193+5837$ & 1998.665 & $R$ & ICCD & - & - & & & & Very bad seeing \\
\hline HD 208132 & $21516+6545$ & 1998.665 & $R$ & ICCD & $147.7 \pm 0.5^{*}$ & $1.450 \pm .002$ & & & & \\
\hline HD 208816 & $21567+6338$ & 1998.665 & $R$ & ICCD & - & - & & & & $\mathrm{SB} \mathrm{P}=7430$ days \\
\hline HD 209790 & $22038+6438$ & 1998.665 & $R$ & ICCD & - & - & & & & $\mathrm{SB} P=811$ days \\
\hline HD 213310 & $22295+4742$ & 1998.657 & $V$ & ICCD & - & - & & & & $\mathrm{SB} \mathrm{P}=42 \mathrm{yr}$ \\
\hline HD 213973 & $22330+6955$ & 1998.665 & $R$ & ICCD & $116.0 \pm 1.0$ & $0.198 \pm .004$ & -3.8 & 0.012 & SJ99 & \\
\hline$"$ & $"$ & 1998.665 & $R$ & ICCD & $121.2 \pm 1.0$ & $0.204 \pm .008$ & 1.4 & 0.018 & SJ99 & \\
\hline HD 214558 & $22383+4511$ & 1998.657 & $V$ & ICCD & - & - & & & & Artefact in central line \\
\hline HD 214606 & $22373+6913$ & 1998.665 & $R$ & ICCD & $0.2 \pm 0.5$ & $0.535 \pm .007$ & & & & $\mathrm{~A}$ is $\mathrm{Am}$, perhaps $\mathrm{SB}$ \\
\hline HD 215242 & $22431+4710$ & & & & & & & & & Triple system \\
\hline$"$ & $\mathrm{AB}$ & 1993.605 & $V$ & CAR & $300.0 \pm 1.0$ & $0.462 \pm .01$ & & & & \\
\hline$"$ & $\mathrm{AC}$ & 1993.605 & $V$ & CAR & $309.0 \pm 1.0$ & $0.450 \pm .01$ & & & & \\
\hline$"$ & $\mathrm{BC}$ & 1993.605 & $V$ & CAR & $42 . \pm 6$ & $0.069 \pm .02$ & & & & \\
\hline$"$ & $\mathrm{AB}$ & 1993.605 & $R$ & CAR & $300.7 \pm 1.0$ & $0.453 \pm .01$ & & & & \\
\hline$"$ & $\mathrm{AC}$ & 1993.605 & $R$ & CAR & $308.5 \pm 1.0$ & $0.453 \pm .01$ & & & & \\
\hline$"$ & $\mathrm{BC}$ & 1993.605 & $R$ & CAR & $34 . \pm 6$ & $0.054 \pm .01$ & & & & \\
\hline$"$ & $\mathrm{AB}$ & 1993.608 & $B$ & CAR & - & - & & & & Marginal detection. \\
\hline$"$ & $\mathrm{AB}$ & 1993.611 & $B$ & CAR & - & - & & & & \\
\hline$"$ & $\mathrm{AB}$ & 1995.553 & $R$ & CAR & - & - & & & & Marginal detection. \\
\hline$"$ & $\mathrm{AB}$ & 1995.556 & $B$ & CAR & $306.0 \pm 1.0$ & $0.517 \pm 0.01$ & & & & \\
\hline$"$ & $\mathrm{AB}$ & 1995.556 & $V$ & CAR & $305.2 \pm 1.0$ & $0.508 \pm 0.01$ & & & & \\
\hline$"$ & $\mathrm{AB}$ & 1995.556 & $R$ & CAR & $305.7 \pm 1.0$ & $0.499 \pm 0.01$ & & & & \\
\hline$"$ & $\mathrm{AB}$ & 1997.476 & $R$ & PAPA & $302.8 \pm 1.0$ & $0.509 \pm .01$ & & & & \\
\hline$"$ & $\mathrm{AC}$ & 1997.476 & $R$ & PAPA & $308.2 \pm 1.0$ & $0.507 \pm .01$ & & & & \\
\hline$"$ & $\mathrm{BC}$ & 1997.476 & $R$ & PAPA & $44.0 \pm 5$ & $0.056 \pm .01$ & & & & \\
\hline$"$ & $\mathrm{AB}$ & 1998.657 & $B$ & ICCD & $302.6 \pm 1.5$ & $0.496 \pm .005$ & & & & Fuzzy autocorr. peaks \\
\hline$"$ & $\mathrm{AB}$ & 1998.657 & $V$ & ICCD & $304.6 \pm 0.5$ & $0.501 \pm .002$ & & & & \\
\hline$"$ & $\mathrm{AB}$ & 1998.657 & $R$ & ICCD & $304.4 \pm 0.5^{*}$ & $0.499 \pm .002$ & & & & \\
\hline$"$ & $\mathrm{AB}$ & 1998.660 & $V$ & ICCD & $304.5 \pm 0.5^{*}$ & $0.499 \pm .002$ & & & & \\
\hline HD 223047 & $23460+4625$ & 1995.550 & $R$ & CAR & - & - & & & & $\psi$ And; Marginal detection. \\
\hline$"$ & $"$ & 1995.556 & $B$ & CAR & $282.0 \pm 1.7$ & $0.327 \pm .009$ & & & & \\
\hline$"$ & $"$ & 1995.559 & $B$ & CAR & $282.3 \pm 0.7$ & $0.325 \pm .006$ & & & & \\
\hline$"$ & $"$ & 1995.559 & $V$ & CAR & $281.6 \pm 1.0$ & $0.317 \pm .004$ & & & & \\
\hline$"$ & $"$ & 1998.657 & $B$ & ICCD & $280.1 \pm 0.5$ & $0.351 \pm .008$ & & & & Artefact in central line \\
\hline$"$ & $"$ & 1998.657 & $V$ & ICCD & $280.3 \pm 0.5$ & $0.332 \pm .009$ & & & & \\
\hline$"$ & $"$ & 1998.657 & $R$ & ICCD & $280.2 \pm 0.5^{*}$ & $0.342 \pm .005$ & & & & \\
\hline
\end{tabular}


The smallest (one-sigma) errors for the angular separation (Col. 6) were estimated at $0.002^{\prime \prime}$ for close pairs (i.e., $\rho<1^{\prime \prime}$ ) and $0.2 \%$ for wide pairs (i.e., $\rho>1^{\prime \prime}$ ) on the basis of the uncertainties coming from the determination of the center of the autocorrelation peak and those affecting scale calibration. Similarly, the smallest (one-sigma) error found for the angle position (Col. 7) was $0.5^{\circ}$.

To evaluate systematic errors (e.g. a wrong value of the focal length), we plotted in Fig. 1 the residuals relative to the ephemerides for objects with known orbits (Cols. 8 and 9 of Tables 3 and 4). In this plot, we excluded the case of HD 196088 whose latest orbit lead to particularly large residuals (discussed in Sect. 5.1). Although some residuals are rather large - due to the poor knowledge of the orbits for these objects —, they are globally centered at $(\Delta \theta=0 ; \Delta \rho=0)$, which indicates the absence of systematic errors.

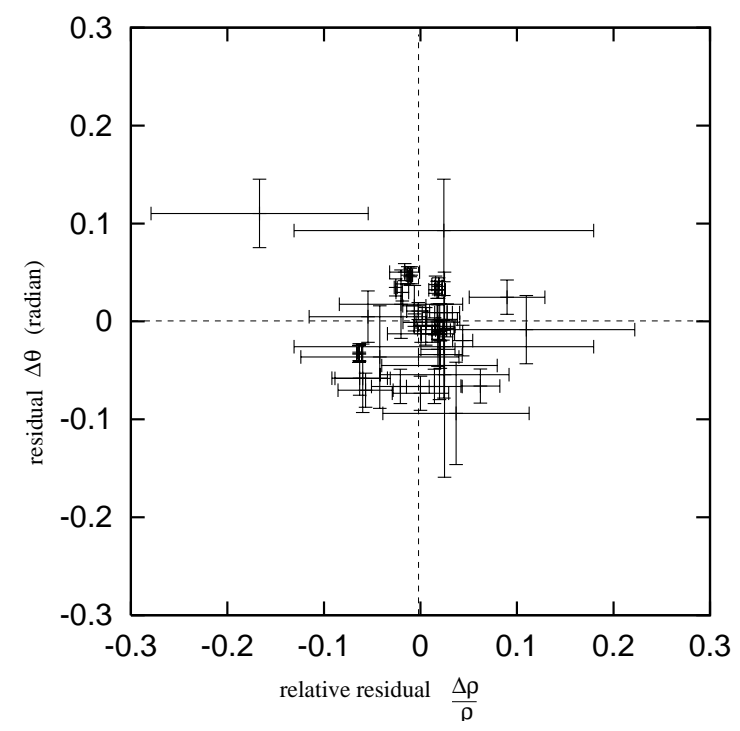

Fig. 1.- Residuals for objects with known orbits (observed - computed).

Table 3-Continued

\begin{tabular}{ccccccccccc}
\hline \hline HD name & WDS name & Epoch & Filt. Detector & $\begin{array}{c}\rho \\
\circ\end{array}$ & $\begin{array}{c}\rho \\
\prime \prime\end{array}$ & $\begin{array}{c}\theta_{(O-C)} \\
\circ\end{array}$ & $\begin{array}{c}\rho_{(O-C)} \\
\prime \prime\end{array}$ & Orbit & Comments \\
$(1)$ & $(2)$ & $(3)$ & $(4)$ & $(5)$ & $(6)$ & $(7)$ & $(8)$ & $(9)$ & $(10)$ & $(11)$
\end{tabular}

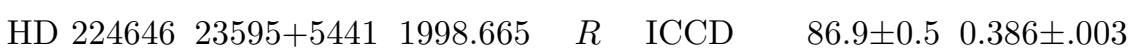


Table 4. Relative astrometry of our complementary list (same structure as Table 3).

\begin{tabular}{|c|c|c|c|c|c|c|c|c|c|c|}
\hline HD name & $\begin{array}{c}\text { WDS name } \\
\text { (2) }\end{array}$ & $\begin{array}{c}\text { Epoch } \\
(3)\end{array}$ & $\begin{array}{l}\text { Filt. } \\
(4)\end{array}$ & Detector & $\begin{array}{c}\theta \\
\circ \\
(6)\end{array}$ & $\begin{array}{c}\rho \\
\prime \prime \\
(7)\end{array}$ & $\begin{array}{c}\theta_{(O-C)} \\
\circ \\
(8)\end{array}$ & $\begin{array}{c}\rho_{(O-C)} \\
\quad{ }^{\prime \prime} \\
(9)\end{array}$ & $(10)$ & $\begin{array}{l}\text { Comments } \\
\text { (11) }\end{array}$ \\
\hline ADS 9701 & $15348+1032$ & 1995.556 & $B$ & CAR & $174.8 \pm 0.5^{*}$ & $4.00 \pm .01$ & -1.8 & -0.254 & HP73 & \\
\hline$"$ & $"$ & 1995.556 & $V$ & CAR & $174.7 \pm 0.5^{*}$ & $4.00 \pm .01$ & -1.9 & -0.254 & HP73 & \\
\hline$"$ & $"$ & 1995.556 & $R$ & CAR & $174.7 \pm 0.5^{*}$ & $4.00 \pm .01$ & -1.9 & -0.254 & HP73 & \\
\hline ADS 11046 & $18055+0230$ & 1995.559 & $B$ & CAR & $165.7 \pm 0.5^{*}$ & $2.63 \pm .02$ & -0.4 & 0.071 & PB00 & \\
\hline$"$ & $"$ & 1995.559 & $V$ & CAR & $165.6 \pm 0.6^{*}$ & $2.62 \pm .02$ & -0.5 & 0.061 & PB00 & \\
\hline$"$ & $"$ & 1995.559 & $R$ & CAR & $165.6 \pm 0.6^{*}$ & $2.61 \pm .02$ & -0.5 & 0.051 & PB00 & \\
\hline HD 895 & $00134+2659$ & 1993.602 & $V$ & CAR & $172.5 \pm 1$ & $0.347 \pm .01$ & -3.8 & 0.005 & $\mathrm{SC00}$ & \\
\hline$"$ & $"$ & 1993.608 & $V$ & CAR & $172.1 \pm 1$ & $0.342 \pm .01$ & -4.2 & 0.000 & $\mathrm{SC} 00$ & \\
\hline$"$ & $"$ & 1993.608 & $R$ & CAR & $172.5 \pm 1$ & $0.335 \pm .01$ & -3.8 & -0.007 & $\mathrm{SC} 00$ & \\
\hline HD 2913 & $00324+0657$ & 1993.614 & $B$ & CAR & - & $0.180 \pm .01$ & & & & \\
\hline$"$ & $"$ & 1993.611 & $V$ & CAR & $286.7 \pm 3$ & $0.198 \pm .015$ & -5.4 & 0.007 & MS97 & \\
\hline$"$ & $"$ & 1993.611 & $V$ & CAR & $290.0 \pm 3$ & $0.183 \pm .015$ & -2.1 & -0.008 & MS97 & \\
\hline HD 156729 & $17177+3717$ & 1994.720 & $R$ & CAR & $139.1 \pm 0.8$ & $0.906 \pm .007$ & & & & \\
\hline HD 196178 & $20339+4642$ & 1993.602 & $V$ & CAR & $60.9 \pm 3$ & $0.260 \pm .02$ & & & & \\
\hline$"$ & $"$ & 1993.605 & $V$ & CAR & $63.0 \pm 2$ & $0.270 \pm .02$ & & & & \\
\hline$"$ & $"$ & 1993.614 & $R$ & CAR & $62.3 \pm 2$ & $0.305 \pm .03$ & & & & \\
\hline HD 197018 & $20396+4035$ & 1993.602 & V & CAR & $1.6 \pm 1$ & $0.871 \pm .02$ & & & & \\
\hline HD 206644 & $21424+4105$ & 1993.602 & $V$ & CAR & $335.5 \pm 2$ & $0.167 \pm .01$ & -2.6 & 0.003 & HK00 & \\
\hline HD 214810 & $22408-0333$ & 1993.614 & $B$ & CAR & $126.2 \pm 2$ & $0.349 \pm .01$ & -3.3 & -0.021 & SJ99 & \\
\hline$"$ & $"$ & 1993.605 & $V$ & CAR & $126.2 \pm 1$ & $0.348 \pm .01$ & -3.3 & -0.022 & SJ99 & \\
\hline$"$ & $"$ & 1993.605 & $R$ & CAR & $125.5 \pm 1$ & $0.350 \pm .01$ & -4.0 & -0.020 & SJ99 & \\
\hline$"$ & $"$ & 1995.550 & $V$ & CAR & - & - & & & & Marginal detection \\
\hline$"$ & $"$ & 1995.550 & $R$ & CAR & $129.0 \pm .9$ & $0.384 \pm .004$ & -1.1 & 0.017 & SJ99 & \\
\hline V Cyg. & - & 1993.605 & $R$ & CAR & - & - & & & & Asymmetric core \\
\hline
\end{tabular}




\section{Discussion}

We compared our measurements with those from other observers, making an extensive use of the CHARA Third Catalogue (http://ad.usno.navy.mil/dsl/Speckle/intro.html, hereafter CHARA3), which includes all published measures of binary stars obtained with interferometric techniques.

\subsection{Composite spectrum stars: particular cases}

In this section, we successively examine the binaries (L 4, WGT 1Aa and BON Ap), whose duplicity is confirmed for the first time since their discovery, and the objects HD 49618 and HD 196088 which exhibit the largest residuals in Table 3 (Cols. 8 and 9).

The companion of HD 29104 (L 4, ADS 3316) was discovered by Thomas Lewis in 1902 with the 28 -inch refractor of the Greenwich Observatory, with $\theta=192^{\circ}$ and $\rho=0.34^{\prime \prime}$ (Dyson, 1921), and was never detected again, despite numerous attempts, especially in the period 1910-1922. Aitken (1932) concluded in his famous "New General Catalogue of Double Stars" by the comment: "It is very doubtful whether the star is double". Only one observation (negative detection) is reported in CHARA3 (Bonneau et al., 1984). Our measurement in 1994.720 with $\theta=134.0^{\circ}$ and $\rho=0.065^{\prime \prime}$ is then the first confirmation of the duplicity of HD 29104, more than 90 years after its discovery.

Likewise, our observations of HD 83808 (WGT 1Aa, ADS 7480) and HD 183912 Aa' (BON Ap) are the first successful detections since the discovery of the companions for both objects, respectively with $\theta=17.0^{\circ}, \rho=0.463^{\prime \prime}$ in 1980 (Weigelt \& Wirnitzer, 1983) and $\theta=163^{\circ}, \rho=0.125^{\prime \prime}$ in 1978 (Bonneau \& Foy, 1980).

For HD 49618 (STF 963 AB, ADS 5514), the orbit computed by Docobo \& Costa (1984) fits better our measurements than Heintz' (1986) orbit, although the residuals are large in both cases. This was unexpected since Heintz' orbit is more recent and should be more accurate.

The $O-C$ residuals of HD 196088 (MCA 61, BD+49 3310, HD 196089) are the largest seen in Fig. 1. This is surprising since the orbit was computed in 2000 (Olevic \& Jovanovic, 2000) and should be compatible with our observation, made in 1998.665. We are quite confident about the quality of our measurement since it is in good agreement with the previous speckle observations reported in CHARA3. The angular separation has been increasing rather regularly from $0.047^{\prime \prime}$ to $0.074^{\prime \prime}$ during the period 1989-1996. Our measurement shows that the periastron passage predicted in 1998.49 by Olevic \& Jovanovic did not occur.

As a general remark, our measurements will be very useful for future orbit determinations, because the number of speckle measurements of composite stars, and of binaries in general, has been decreasing in the 1990's. For example, since the Hipparcos measurement of 1991.25 (ESA, 1987), only one measurement is given in CHARA3 for the objects HD 195692 
(STF 2695, ADS 13971) and HD 214606 (CHR 113, BD+68 1319), obtained by Hartkopf et al. (2000) in 1996 and 1994, respectively. This decrease of the occurrence rate of speckle observations is of particular importance for the small angular separations, due to a lack of observations with large telescopes.

\subsection{Complementary list: particular cases}

For ADS 9701 (WDS 15348+1032), the orbit computed by Hopmann (1973) leads to large residuals $\left(\rho_{(O-C)}=-0.254^{\prime \prime}\right)$ and clearly needs revision. The orbit of this double star is still undetermined because the companion has moved along an orbital arc of only $26^{\circ}$ between 1822 and 1990. The relative motion of the two components is very slow and the period is thus expected to be very large. In the hypothetical case (quite unlikely) of a circular orbit, the orbital period would be about 2300 years. Our measurement is in agreement with the Hipparcos data: $\theta=174.8^{\circ}, \rho=4.012^{\prime \prime}$.

During the data reduction, the objects HD 156729 and HD 196178 that we intended to use as reference stars were subsequently found to be double stars (Table 4).

HD 196178 (HIP 101475, WDS 20339+4642) was discovered as a double system by Hipparcos with $\rho=0.311^{\prime \prime}$ and $\theta=56^{\circ}$. In CHARA3, only one ground-based observation is reported in 1996.699, with $\rho=0.302^{\prime \prime}$ and $\theta=60.2^{\circ}$ (Mason et al., 1999b).

HD 156729 (HR 6436, HIP 84606) was also found double by Hipparcos: $\rho=0.844^{\prime \prime}$, $\theta=141^{\circ}$, with $\Delta m \sim 4.2$. This large magnitude difference between the two components explains why all speckle detection attempts of the companion have failed in 1988.169 ( $\rho<$ 0.038", McAlister et al., 1993), in 1997.269 ( $\rho<0.054^{\prime \prime}$, Mason et al., 1999b) and in 1997.460 $\left(\rho<0.054^{\prime \prime}\right.$, Mason et al., 1999b). Our measurement in $1994.720\left(\rho=0.906^{\prime \prime}, \theta=139^{\circ}\right)$ agrees well with the Hipparcos data. We also found a large magnitude difference, the companion brightness being close to our detection limit.

\subsection{Composite spectrum stars not resolved by PISCO}

We now examine the possible reasons which may account for the non-detection of the companion of some targets. Let us recall that we could not resolve all the binary stars with angular separations less than the TBL diffraction limit $\rho_{d}\left(\rho_{d}=0.05^{\prime \prime}\right.$ in $\left.V\right)$.

The objects that were never resolved during our observations are listed in Table 5. If we take into account the last measurements by other observers (Cols. 2-5), the objects of this table belong to three groups (cf. Col. 6):

(1) binaries with a very small angular separation $\rho<\rho_{d}$,

(2) binaries with $\rho_{d} \leq \rho \leq 2 * \rho_{d}$, that can be resolved when atmospheric conditions are good, 
(3) objects whose duplicity poses problem because it was never confirmed since their discovery.

\subsubsection{Objects with small angular separations}

Among our sample of composite spectrum stars, there are binaries whose components are closer than the TBL diffraction limit over part of their orbit. Hence the absence of a companion detection by PISCO is not surprising. An extreme case is HD 192577 (WRH 33Aa) which was always observed with angular separations smaller than $0.03^{\prime \prime}$.

With angular separations larger than $\rho_{d}$, the objects of the second group should have been resolved by PISCO. Let us remark that all of these objects were only observed once by PISCO and this absence of detection is not meaningful. It must be due to bad seeing. Actually, the performances of speckle interferometry are tightly related to the coherence time $\tau_{c}$ and radius $r_{0}$ of the atmospheric turbulence (cf., e.g., Roddier, 1981). As the turbulence can vary quickly, $\tau_{c}$ and $r_{0}$ can quickly reach values small enough to impede the resolution of close binaries. This explains why in Table 3, there are many negative detection reports associated with positive detections for the same object (e.g., HD 9352, HD 82072, and HD $183912 \mathrm{Aa}$ ), sometimes on the same night.

\subsubsection{Dubious or difficult cases}

There are objects that were never resolved with PISCO at the TBL, despite multiple attempts made during the 1993-1998 period, and whose duplicity has never been confirmed either by other observers since they were discovered:

HD 17245 (CHR 7): 3 observations were made in 1980-1984, with only one positive detection, by the discoverer (McAlister et al., 1987). We observed HD 17245 four times in 1997-1998 without success.

HD 17378 (MCA 9): Only two observations are reported in CHARA3. The companion was found in 1980 by McAlister \& Hartkopf (1984) and was not detected in 1984 with a 6-meter telescope (Balega \& Balega, 1987). Our three observations in 1994-1998 led to a negative result.

HD 109358 (BNU 4Aa): It was discovered as double with $\rho=0.110^{\prime \prime}$ by Bonneau et al. (1980), but was never detected in any of the 17 other observations performed from 1921 to 1989. Our two observations in 1997 also led to a negative result.

HD 169985 (STF 2316 Aa): The companion was observed twice in 1951, with $\rho=0.06^{\prime \prime}$ (Wilson, 1952), and 1976, with $\rho=0.250^{\prime \prime}$ (McAlister \& Hendry, 1982). All 8 subsequent observations in 1976-1992 have failed.

HD 192909 (V1488 Cyg): The 20 observations made during the period 1921-1992 have 
Table 5. Composite spectrum stars not resolved by PISCO.

\begin{tabular}{|c|c|c|c|c|c|}
\hline \multirow[t]{2}{*}{ Name } & \multicolumn{4}{|c|}{ Last companion detection } & \multirow[t]{2}{*}{ Group } \\
\hline & Epoch & $\theta$ (deg.) & $\rho(\operatorname{arcsec})$ & Reference & \\
\hline $\begin{array}{l}\text { HD } 4775=\text { MCA } 2=\text { HR } 233 \\
\text { HD } 192577=\text { WRH } 33 \mathrm{Aa}=\text { ADS } 13554 \\
\text { HD } 184759=\text { WRH } 32=\text { HR } 7441 \\
\text { HD } 209790=\text { MCA } 69 \mathrm{Aa}=\text { ADS } 15600\end{array}$ & $\begin{array}{l}1994.719 \\
1985.843 \\
1994.721 \\
1994.716\end{array}$ & $\begin{array}{c}212.5 \\
111.4 \\
17.8 \\
206.4\end{array}$ & $\begin{array}{l}0.022 \\
0.027 \\
0.032 \\
0.039\end{array}$ & $\begin{array}{l}\text { Schoeller et al. (1998) } \\
\text { McAlister et al. (1987) } \\
\text { Schoeller et al. (1998) } \\
\text { Schoeller et al. (1998) }\end{array}$ & $\begin{array}{c}(1) \\
\rho<0.05^{\prime \prime}\end{array}$ \\
\hline $\begin{array}{l}\text { HD } 13474=\text { MCA } 6=\text { HR } 640 \\
\text { HD } 23089=\text { MCA } 12=\text { HR } 1129 \\
\text { HD } 203338=\text { BAG 9Aa }=\text { ADS } 14864 \\
\text { HD } 213310=\text { MCA } 71=\text { HR } 8572 \\
\text { HD } 214558=\text { CHR } 114=\text { HR } 8617\end{array}$ & $\begin{array}{l}1994.709 \\
1993.205 \\
1996.538 \\
1994.716 \\
1996.553\end{array}$ & $\begin{array}{c}217.8 \\
359.2 \\
121.8 \\
43.1 \\
205.5\end{array}$ & $\begin{array}{l}0.07 \\
0.054 \\
0.112 \\
0.082 \\
0.114\end{array}$ & $\begin{array}{l}\text { Hartkopf et al. (2000) } \\
\text { Hartkopf et al. (1994) } \\
\text { Hartkopf et al. (2000) } \\
\text { Hartkopf et al. (1997) } \\
\text { Hartkopf et al. (2000) }\end{array}$ & $\begin{array}{c}(2) \\
0.05^{\prime \prime} \leq \rho \leq 0.1^{\prime \prime}\end{array}$ \\
\hline $\begin{array}{l}\text { HD } 17245=\mathrm{CHR} 7=\mathrm{BD}+43576 \\
\text { HD } 17378=\mathrm{MCA} 9=\mathrm{HR} 825 \\
\text { HD } 109358=\mathrm{BNU} 4 \mathrm{Aa}=\mathrm{HR} 4785 \\
\text { HD } 169985=\mathrm{STF} 2316 \mathrm{Aa}=\mathrm{ADS} 11353 \\
\text { HD } 192909=\mathrm{V} 1488 \mathrm{Cyg}=\mathrm{HR} 7751 \\
\text { HD } 197177=\mathrm{BNU} 7 \mathrm{Aa}=\mathrm{ADS} 14158 \\
\text { HD } 208816=\mathrm{WRH} 36=\mathrm{HR} 8383\end{array}$ & $\begin{array}{c}1984.058 \\
1980.893 \\
1978.397 \\
1976.613 \\
- \\
1979.463 \\
1950.560\end{array}$ & $\begin{array}{c}104.4 \\
98.7 \\
43 \\
156.9 \\
- \\
19.0 \\
174.5\end{array}$ & $\begin{array}{c}0.161 \\
0.186 \\
0.110 \\
0.250 \\
- \\
0.244 \\
0.05\end{array}$ & \begin{tabular}{c|} 
McAlister et al. (1987) \\
McAlister \& Hartkopf (1984) \\
Bonneau et al. (1980) \\
McAlister \& Hendry (1982) \\
Never resolved \\
Bonneau et al. (1980) \\
Wilson (1951)
\end{tabular} & $\begin{array}{l}\text { (3) } \\
\text { Dubious or } \\
\text { difficult cases }\end{array}$ \\
\hline
\end{tabular}


failed to detect the companion.

HD 197177 (BNU 7Aa): The companion was only observed once by the discoverers with $\rho=0.244^{\prime \prime}$ (Bonneau et al., 1980). Nine other detection attempts made in 1949-1986 led to negative results.

HD 208816 (WRH 36): The companion was only seen by Wilson (1951), with a very small separation: $\rho=0.05^{\prime \prime}$, and never confirmed despite 17 other attempts in 1949-1992.

Hence, in the last twenty years or so, all the efforts made to resolve these objects with speckle interferometry have failed. One can then wonder whether the companions are detectable by speckle interferometry. As the spectra of all these objects are composite, their binary nature is certain and the magnitude difference between their two components is not very large $(\leq 3)$. Hence the non-detection of the companion by speckle interferometry likely means that the two components are closer than the diffraction limit of the telescopes used.

\section{Conclusion}

The PISCO speckle camera of the Midi-Pyrénées Observatory has proven to be an efficient tool to study binary and multiple stars. Despite bad weather conditions, campaigns led with PISCO have produced a large number of astrometric measurements of binary systems, many of which could be followed during six years.

Three very different detectors were used (CAR, ICCD, PAPA), with different processing procedures. However, the results are homogeneous. The very good agreement both internally - measurements obtained with those three detectors agree - and externally - agreement with speckle interferometry observations by other authors - shows the absence of systematic errors when operating PISCO.

We confirm the duplicity of HD 156729 (HR 6436) discovered by Hipparcos as having a large magnitude difference between the two components $(\Delta m=4.2)$. PISCO is the first ground-based instrument that has been able to resolve this difficult object. Indeed, in most books, it is said that the speckle technique is limited to $\Delta m \leq 3$. The CAR detector, that was used for this detection, appears then particularly sensitive, and exhibits a high dynamical range. We also confirm the duplicity of HD 29104 (L 4), HD 83808 (WGT 1Aa), and HD 183912 Aa' (BON Ap). For these objects, the measurements with PISCO are the first successfully performed since the discovery of their duplicity, in 1902, 1980 and 1978, respectively.

Some objects were not resolved during our observations. A close analysis of the results obtained by previous observers yields a logical explanation. For most objects, the angular separation was very close to, or smaller than, the diffraction limit of the TBL whose diameter is too small to perform continuous measurements during an orbital revolution. For others, the companion was observed only once, by their discoverers. As the presence of these companions 
has never been confirmed in the last 20 years or so, even with large telescopes, the two components responsible for the composite spectrum of these objects must be too close to be detected by 2-6 m class telescopes. To resolve them, interferometric arrays are required. Since these objects are very close binaries whose components are rather bright and have a small magnitude difference, they should be useful for testing the capabilities of these arrays.

Due to a change in policy of the TBL time committee, PISCO was de-commissioned in 1998 and this paper is the last of a series that has produced numerous results (Carbillet et al. 1996;

Aristidi et al. 1997b; Aristidi et al. 1999; 48; 45). PISCO has contributed measurements of binary stars during the particularly critical period of the 1990's during which the frequency of speckle measurements with 2-m class, or larger, telescopes significantly decreased. Continuous observations are essential for establishing reliable orbits. In particular, measurements obtained during the periastron passage are crucial for accurate orbit determinations. The case of HD 196088 is illustrative of this problem. Our observations obtained close to the predicted periastron invalidate a very recently published orbit. This erroneous orbit was computed after the epoch of our observations, using too scarcely distributed data. We hope the situation will soon improve with new generation speckle cameras being installed on large-size telescopes and adaptive optics systems becoming available on most of medium-size telescopes.

We would like to thank the technical staff of the "Télescope Bernard Lyot" for providing assistance during the observations.

We are grateful to the people who lent us some of the detectors used: Peter Nisenson (Center for Astrophysics, USA) for the PAPA detector and Denis Mourard (Côte d'Azur Observatory, France) for the CAR detector. We also thank Jean-Pierre Barriot (Midi-Pyrénées Observatory), Yves Bresson (Côte d'Azur Observatory), Christine André, Boris Dintrans and Pierre-Antoine Hermange (all students at Midi-Pyrénées Observatory), and Bruno Lopez (Côte d'Azur Observatory) who participated to some of the observations.

This work has made use of the "Third Catalog of Interferometric Measurements of Binary Stars" (http://ad.usno.navy.mil/dsl/Speckle/intro.html, CHARA Contribution No4) and of the "Besançon data base of binary stars" (http://bdb.obs-besancon.fr). This research has also made use of the Washington Double Star Catalog maintained at the U.S. Naval Observatory.

\section{REFERENCES}

Aitken R.G., 1932, "New General Catalogue of Double Stars", Carnegie institution, Washington.

Aristidi E., Carbillet M., Lyon J.-F., Aime C., 1997a, A\&AS, 125, 13.

Aristidi E., Carbillet M., Prieur J.-L, Lopez B., Bresson Y., Koechlin L., 1997b, A\&AS, 126, 555. 
Aristidi E., Prieur J.-L., Scardia M., Koechlin L., Avila R., Lopez B., Rabbia Y., Carbillet M., Nisenson P., Gezari D., 1999, A\&AS, 134, 545.

Balega I.I., Balega Y.Y., 1987, SvAL 13, 208.

Balega I.I., Balega, Y.Y., Maksimov A.F., Pluzhnik E.A., Shkhagosheva Z.U., Vasyuk V.A., 1999, A\&AS 140, 287.

Bonneau D., Foy R., 1980, A\&A 86, 295.

Bonneau D., Blazit A., Foy R., Labeyrie A., 1980, A\&AS 42, 185.

Bonneau D., Carquillat J.M., Vidal J.L., 1984, A\&AS 58, 729.

Carbillet M., Lopez B., Aristidi E., Bresson Y., Aime C., Ricort G., Prieur J.-L., Koechlin L., Helmer G., Lefèvre J., Cruzalebes P., 1996, A\&A, 314, 112.

Cuypers J., \& Seggewiss W., 1999, A\&AS, 139, 425.

Docobo J.A., Costa J.M., 1984, IAU Comm. 26, Inf. Circ. 93.

Dyson F.W., 1921, "Catalogue of double stars from observations made at the Royal Observatory, Greenwich, with the 28 inch refractor during the years 1893-1919", H. M. Stationery Office, London.

ESA, 1987, Hipparcos Catalog, ESA SP-1200,

Ginestet N., Carquillat J.-M., Jaschek M., Jaschek C., Pédoussaut A., Rochette J., 1992, Atlas de spectres stellaires, Obs. Midi-Pyrénées, Obs. de Strasbourg (France).

Ginestet N., Carquillat J.-M., Jaschek C., Jaschek M., 1997, A\&AS, 123, 135.

Ginestet N., Carquillat J.-M., Jaschek C., 1999, A\&AS, 134, 473.

Griffin R.F., 1990, JA\&A, 11, 491.

Hartkopf W.I., McAlister H.A., Franz O.G., 1989, AJ. 98, 1014.

Hartkopf W.I., McAlister H.A., Mason B.D., Barry D.J., Turner N.H., Fu H.-H. 1994, AJ, $108,2299$.

Hartkopf W.I., McAlister H.A., Mason B.D., ten Brummelaar T.A., Roberts J.C. Jr., Turner N.H., Wilson J.W., 1997, AJ, 114, 1639.

Hartkopf W.I., 1999, IAU Comm. 26, Inf. Circ. 139.

Hartkopf W.I., Mason B.D., McAlister H.A., Roberts L.C. Jr., Turner N.H., ten Brummelaar T.A., Prieto C.M., Ling J.F., Franz O.G., 2000, AJ 119, 3084.

Hartkopf W.I., Mason B.D., 2000, IAU Comm. 26, Inf. Circ. 142. 
Heintz W.D., 1982, A\&AS 47, 569.

Heintz W.D., 1986, A\&AS 64, 1.

Hoffleit D., Jaschek C., 1982, "The Bright Star Catalogue", Yale Univ. Obs., New Haven.

Hopmann J., 1964, Ann. Sternw. Wien 26, 28.

Hopmann J., 1973, Mitt. Wien n. 13.

Hynek J.A. 1938, Contr. Perkins Obs. No 10.

Markowitz A.H., 1969, Ph.D. Thesis, Ohio State University.

Mason B.D., 1997, AJ 114, 808, 1997.

Mason B.D., Douglass G.G., Hartkopf W.I., 1999a, AJ 117, 1023.

Mason B.D., Martin C., Hartkopf W.I., Barry D.J., Germain M.E., Douglass, G.G., Worley, C.E., Brummelaar T.A., Franz O.G., 1999b, AJ, 117, 1890.

McAlister H.A., Hendry E.M., 1982, ApJS 48, 273.

McAlister H.A., Hartkopf W.I. 1984, Catalog of Interferometric Measurements of Binary Stars, CHARA Contrib. No. 1.

McAlister H.A., Hartkopf W.I., Hutter D.J., Franz O.G., 1987, AJ 93, 688

McAlister H.A., Hartkopf W.I., 1988, Second Catalog of Interferometric Measurements of Binary Stars, CHARA contr. No 2.

McAlister H.A., Mason B.D., Hartkopf W.I., Shara M.M., 1993, AJ, 106, 1639.

Olevic D.J., Jovanovic P., 2000, IAU Comm. 26, Inf. Circ. 141.

Papaliolios C., Nisenson P., Ebstein S., 1985, Appl. Optics, 24, 287.

Pourbaix D., 2000, A\&AS 145, 215.

Prieur J.-L., Lannes A., Cullum M., 1991, Proc. of the ESO conf. High Resolution Imaging by interferometry II, (Ed. J.M. Beckers and F. Merckle) Garching, Germany, 353.

Prieur J.-L, Koechlin L., André C., Gallou G., Lucuix C., 1998, Exper. Astron., 8, 297.

Prieur J.-L., Oblak E., Lampens P., Aristidi E., Kurpinska-Winiarska M., Koechlin L., Ruymaekers G., 2001, A\&A, 367, 865.

Roddier F., 1981, "The effects of atmospheric turbulence in optical astronomy", Ed. E. Wolf, Progress in optics XIX North-Holland, Vol. 2, 283.

Scardia M., 1983, Astron. Nachr. 304, 257. 
Scardia M., Prieur J.-L., Aristidi E., Koechlin L., 2000a, ApJS, 131, 561.

Scardia M., Prieur J.-L., Aristidi E., Koechlin L., 2000b, Astron. Nachr., 321, 4, 255.

Schoeller M., Balega I.I., Balega Y.Y., Hofmann K.-H., Reinheimer T., Weigelt G., 1998, Astron. Lett., 24, 283.

Schröder K.-P., Pols O.R., Eggleton P.P., 1997, MNRAS, 285, 696.

Soderhjelm S., 1999, A\&A 341, 121.

Weigelt G., Wirnitzer B., 1983, Optics Lett 8, 389.

Wilson R.H., 1951, AJ 56, 69.

Wilson R.H., 1952, AJ 57, 248.

Worden S.P., Murray K.S., Schmidt G.D., Angel J.R.P., 1977, Icarus 32, 450. 